\title{
STUDIES ON THE IMPLEMENTATION OF NORMALS AND CURVATURES I. THE FIRST OR MEAN CURVATURE
}

\author{
Carl Rosenkilde \\ Weapons Effects Group \\ D-Division
}

July 2, 1993

This is an informal report intended primarily for internal or limited external distribution. The opinions and conclusions stated are those of the author and may or may not be those of the Laboratory.

Work performed under the auspices of the U.S. Department of Energy by the

Lawrence Livermore National Laboratory under Contract W-7405-Eng-48.

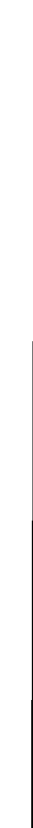

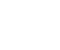

\section{Pt 1}




\section{DISCLAIMER}

This document was prepared as an account of work sponsored by an agency of the United States Government. Neither the United States Government nor the University of California nor any of their employees, makes any warranty, express or implied, or assumes any legal liability or responsibility for the accuracy, completeness, or usefulness of any information, apparatus, product, or process disclosed, or represents that its use would not infringe privately owned rights. Reference herein to any specific commercial products, process, or service by trade name, trademark, manufacturer, or otherwise, does not necessarily constitute or imply its endorsement, recommendation, or favoring by the United States Government or the University of California. The view's and opinions of authors expressed herein do not necessarily state or reflect those of the United States Government or the University of California, and shall not be used for advertising or product endorsement purposes.

This report has been reproduced directly from the best available copy.

Available to DOE and DOE contractors from the Office of Scientific and Technical Information P.O. Box 62, Oak Ridge, TN 37831

Prices available from (615) 576-8401, FTS 626-8401

Available to the public from the National Technical Information Service

U.S. Department of Commerce 5285 Port Royal Rd., Springfield, VA 22161 


\title{
Studies on the Implementation of Normals and Curvatures I.
}

\author{
The First or Mean Curvature
}

We are interested in the construction of the unit normal and the curvatures on some surface which is represented by finite elements. We shall start with the usual definitions for the unit normal and the first and second curvatures on a continuous surface. The unit normal on some surface defined by $\varphi(x)=0$ is given by $\hat{\mathbf{n}}=\nabla \phi / \nabla \phi \mid$, where $\nabla$ signifies the familiar three-dimensional gradient operator in space, rather than the two-dimensional gradient on the a surface, which is commonly utilized in differential geometry. The first and second curvatures, $J$ and $K$, which involve invariant functions of this normal, may be expressed 1 in the form:

$$
2 J=\nabla \cdot \hat{\mathbf{n}} \text { and } 2 K=\nabla \cdot[\hat{\mathbf{n}}(\boldsymbol{\nabla} \cdot \hat{\mathbf{n}})+\hat{\mathbf{n}} \times(\boldsymbol{\nabla} \times \hat{\mathbf{n}})] .
$$

The expression for $J$ has the same formal appearance regardless of which gradient operator is used, but the expression for $K$ differs from the two-dimensional version because of the expansion for the Laplacian of a vector.

Also recall the familiar definitions from vector analysis ${ }^{2}$ for the gradient and the divergence that follow from the divergence theorem when expressed in terms of the limit of the associated surface integral as the enclosed volume goes to zero:

$$
\begin{gathered}
\nabla \varphi=\lim _{V \rightarrow 0} \frac{1}{V} \oiint_{\partial V} d \mathbf{S} \varphi=\lim _{V \rightarrow 0} \frac{1}{V} \oiint_{\partial V} d \mathbf{S n \hat { n }} \varphi \\
\boldsymbol{\nabla} \cdot \mathbf{A}=\lim _{V \rightarrow 0} \frac{1}{V} \oiint_{\partial V} d \mathbf{S} \cdot \mathbf{A}=\lim _{V \rightarrow 0} \frac{1}{V} \oiint_{\partial V} d \mathbf{S} \hat{\mathbf{n}} \cdot \mathbf{A},
\end{gathered}
$$

where $\mathbf{A}$ is an arbitrary vector. In the present case we shall replace $\mathbf{A}$ by $\hat{\mathbf{n}}$ to get the general expressions:

$$
2 J=\lim _{V \rightarrow 0} \frac{1}{V} \oiint_{\partial V} d S \text { and } K=\lim _{V \rightarrow 0} \frac{1}{V} \oiint_{\partial V} J d S .
$$

Let us illustrate these expressions for the case of a simple spherical surface of radius $R$. Choose a thin spherical cap of thickness $\delta R$ which subtends a solid angle $\delta \Omega$. Then the ratio

$$
\frac{1}{V} \oiint_{\partial V} d S=\frac{(R+\delta R)^{2} d \Omega-R^{2} d \Omega}{\frac{1}{3}\left[(R+\delta R)^{3} d \Omega-R^{3} d \Omega\right]}=\frac{2}{R}+O(\delta R)
$$


leads to the expected result and since $\nabla \cdot \hat{\mathbf{n}}=2 / R$ on the surface, inclusion of this factor in the above integral leads to $K=1 / R^{2}$. It is to be noted that in the expansion of the above ratio, the thickness $\delta R$ cancels out of the leading term leaving the desired limiting value.

\section{An Appropriate Unit Normal}

The first step is the construction / specification of unit normals at each nodal point on a surface. Let $m(m \geq 3)$ nodal points surround some specific nodal point, 0 , located on a surface. Figure 1 illustrates a representative configuration consisting of 5 neighboring nodes. Let us denote the

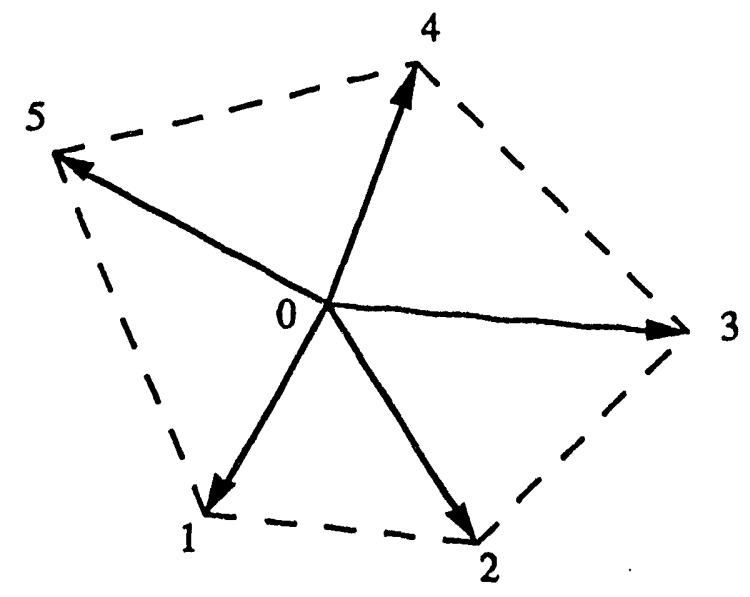

Fig. 1. Representative neighboring surface nodes surrounding a particular surface node.

directions of the element edges emanating from the central node by the vectors $\mathbf{a}_{i}$ and let the normal direction associated with each of these vertices be denoted by the vector cross-product, $\mathbf{a}_{i} \times \mathbf{a}_{i+1}$. Since the area of the associated triangle is half the magnitude of this cross product, it follows that the vectorial surface element associated with each triangular patch takes the form:

$$
\Delta \mathbf{S}_{i}=\hat{\mathbf{n}}_{i} \Delta \mathbf{S} \approx \frac{\mathbf{a}_{i} \times \mathbf{a}_{i+1}}{\left|\mathbf{a}_{i} \times \mathbf{a}_{i+1}\right|} \frac{1}{2}\left|\mathbf{a}_{i} \times \mathbf{a}_{i+1}\right|=\frac{1}{2} \mathbf{a}_{i} \times \mathbf{a}_{i+1} .
$$

(Notice that there is no explicit requirement that the side opposite each vertex be the edge of a finite element.) By summing over the $m$ contiguous triangular patches surrounding the central node, the following approximation may be constructed for the surface integral:

$$
\iint d \mathbf{S} \approx \frac{1}{2} \sum_{i=1}^{m} \mathbf{a}_{i} \times \mathbf{a}_{i+1}=\frac{1}{2} \mathbf{e}_{0}
$$


This construction associates a unique direction with this node. The procedure can be repeated for each node on the surface in order to create a set of unique unit vectors, $\hat{\mathbf{e}}_{i}$, at each surface node.

No volume was utilized in this particular construction, although a weighting with respect to area was introduced explicitly. This particular construction was chosen because it mirrors the above integral expression for the gradient of a scalar function. The associated volume is not required because the resulting vector is to be normalized. However, there are other ways to construct a vector direction associated with each nodal point. For example, one could normalize the vectors, $a_{i}$, at the outset and then form

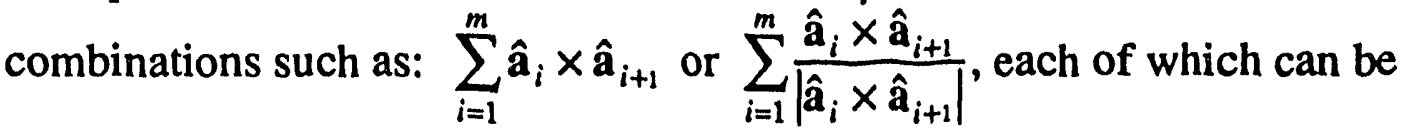
normalized in the usual manner. The first of these utilizes as a weighting factor, an area equal to the sine of the intervening angle; the second simply is a sum of the unit normals associated with each of the individual elements. Neither of these seems to fit into a methodology based upon the above expressions from elementary vector analysis.

Is the unit vector, $\hat{\mathbf{e}}_{0}$, related to the unit outward normal to the surface at this node? Suppose we arbitrarily place the central node (which is labeled by 0 in Fig. 1) on the polar axis passing through a sphere of radius, $R$. Denote the unit vector in this direction as $\hat{\mathbf{e}}_{\mathbf{z}}$ and denote the radial unit vectors at the surrounding nodes by $\hat{\mathbf{r}}_{i}$. Then it follows that the vector

$$
\begin{array}{r}
\mathbf{e}_{0}=\sum_{i=1}^{m} \mathbf{a}_{i} \times \mathbf{a}_{i+1}=R^{2} \sum_{i=1}^{m}\left(\hat{\mathbf{r}}_{i}-\hat{\mathbf{e}}_{\mathbf{z}}\right) \times\left(\hat{\mathbf{r}}_{i+1}-\hat{\mathbf{e}}_{\mathbf{z}}\right) \\
=R^{2} \sum_{i=1}^{m}\left[\hat{\mathbf{r}}_{i} \times \hat{\mathbf{r}}_{i+1}+\hat{\mathbf{e}}_{\mathbf{z}} \times\left(\hat{\mathbf{r}}_{i}-\hat{\mathbf{r}}_{i+1}\right)\right]=R^{2} \sum_{i=1}^{m} \hat{\mathbf{r}}_{i} \times \hat{\mathbf{r}}_{i+1} .
\end{array}
$$

The summation involving $\hat{\mathbf{r}}_{i}-\hat{\mathbf{r}}_{i+1}$ is cyclic since $\hat{\mathbf{r}}_{m+1}=\hat{\mathbf{r}}_{1}$ and sums to zero. The remaining vector will be parallel to $\hat{\mathbf{e}}_{\mathrm{z}}$ if $\hat{\mathbf{e}}_{\mathrm{z}} \times \mathbf{e}_{0}$ vanishes. So, let us expand this triple vector product to get the expression:

$$
\hat{\mathbf{e}}_{\mathrm{z}} \times \mathbf{e}_{0}=R^{2} \sum_{i=1}^{m}\left[\hat{\mathbf{r}}_{i}\left(\hat{\mathbf{e}}_{\mathrm{z}} \cdot \hat{\mathbf{r}}_{i+1}\right)-\hat{\mathbf{r}}_{i+1}\left(\hat{\mathbf{e}}_{\mathrm{z}} \cdot \hat{\mathbf{r}}_{i}\right)\right] \text {. }
$$

This quantity will vanish if all of the dot products have the same value, which means geometrically that all of the vectors $a_{i}$ have the same length and, therefore, end on a circle centered on the central node. In this special case, the vector, $\hat{\mathbf{e}}_{0}$, is identical with $\hat{\mathbf{e}}_{\mathbf{z}}$, which is the unit outward normal 
at that point. We also notice in passing that the above final expression for $\hat{\mathbf{e}}_{0}$ does not depend explicitly upon $\hat{\mathbf{e}}_{z}$, but only upon the directions, $\hat{\mathbf{r}}_{i}$, at the end points of the $a_{i}$ 's. Consequently, the location of the central node can be displaced arbitrarily without affecting the value nor the direction of $e_{0}$ on the spherical surface. We shall return to this point later.

\section{The First or Mean Curvature}

Now let us turn to the estimation of curvature at the central node. We would like to utilize the aforementioned limiting integral relationships from vector analysis. In order to do so we must imagine the construction of a small "pillbox" whose volume can be made to vanish, leaving a non zero limiting value for its ratio with the integral over its surface. Since unique unit vectors, $\hat{\mathbf{e}}_{i}$, can to constructed at each node, let these vectors coincide with the edges of a triangular volume represented in Fig. 2a. Let us suppose that the lengths

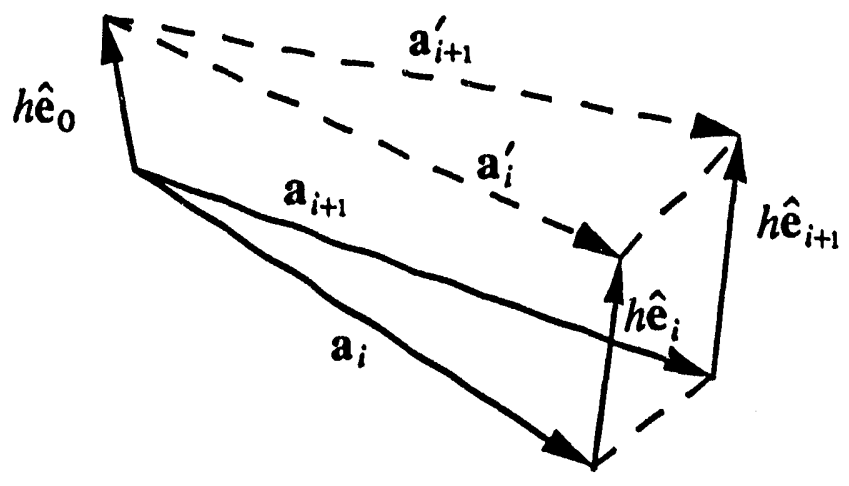

Fig. 2a. The "pillbox" volume.

of these edges are all the same and have the value, $h$. The normal associated with the set of displaced surface elements, whose edges are labeled by primes, is in the direction of

$$
\begin{aligned}
& \mathbf{e}_{0}^{\prime}=\sum_{i=1}^{m} \mathbf{a}_{i}^{\prime} \times \mathbf{a}_{i+1}^{\prime}=\sum_{i=1}^{m}\left\{\left[\mathbf{a}_{i}+h\left(\hat{\mathbf{e}}_{i}-\hat{\mathbf{e}}_{0}\right)\right] \times\left[\mathbf{a}_{i+1}+h\left(\hat{\mathbf{e}}_{i+1}-\hat{\mathbf{e}}_{0}\right)\right]\right\} \\
& =\sum_{i=1}^{m}\left\{\mathbf{a}_{i} \times \mathbf{a}_{i+1}+h\left[\hat{\mathbf{e}}_{i} \times \mathbf{a}_{i+1}+\mathbf{a}_{i} \times \hat{\mathbf{e}}_{i+1}+\hat{\mathbf{e}}_{0} \times\left(\mathbf{a}_{i}-\mathbf{a}_{i+1}\right)\right]+O\left(h^{2}\right)\right\} \\
& =\mathbf{e}_{0}+h \sum_{i=1}^{m}\left(\hat{\mathbf{e}}_{i} \times \mathbf{a}_{i+1}+\mathbf{a}_{i} \times \hat{\mathbf{e}}_{i+1}\right)+O\left(h^{2}\right) .
\end{aligned}
$$


In arriving at this concise form, we have utilized the fact that the cyclic summation, $\sum_{i=1}^{m}\left[\hat{\mathbf{e}}_{0} \times\left(\mathbf{a}_{i}-\mathbf{a}_{i+1}\right)\right]$ over all the nodes surrounding the central node, vanishes. Similarly, the summation, $\sum_{i=1}^{m}\left[\hat{\mathbf{e}}_{i+1} \times \mathbf{a}_{i+1}-\hat{\mathbf{e}}_{i} \times \mathbf{a}_{i}\right]$, also vanishes for the same reason. By adding these two sums back into the above expression, we can obtain yet another interesting form:

$$
\mathbf{e}_{0}^{\prime}=\mathbf{e}_{0}+h \sum_{i=1}^{m}\left[\left(\hat{\mathbf{e}}_{0}+\hat{\mathbf{e}}_{i}+\hat{\mathbf{e}}_{i+1}\right) \times\left(\mathbf{a}_{i+1}-\mathbf{a}_{i}\right)\right]+O\left(h^{2}\right),
$$

which will be helpful later in interpretations.

According to the integral expression for $J$, we need to form the integral of the dot product between to surface elements and the representative unit outward normal. On this account the contributions from the surface areas of the contiguous pillboxes, which surround the central node, are given approximately by :

$$
\begin{aligned}
\oiint_{\delta V} d \mathbf{S} \cdot \hat{\mathbf{n}} & =\iint_{\text {lop }} d \mathbf{S}^{\prime} \cdot \hat{\mathbf{e}}_{0}^{\prime}+\iint_{\text {base }} d \mathbf{S} \cdot \hat{\mathbf{e}}_{0}+O\left(h^{2}\right) \\
& \approx\left(\frac{1}{2} \sum_{i=1}^{m} \mathbf{a}_{i}^{\prime} \times \mathbf{a}_{i+1}^{\prime}\right) \cdot \hat{\mathbf{e}}^{\prime}+\left(-\frac{1}{2} \sum_{i=1}^{m} \mathbf{a}_{i} \times \mathbf{a}_{i+1}\right) \cdot \hat{\mathbf{e}}_{0}+O\left(h^{2}\right) \\
& =\frac{1}{2}\left(\mathbf{e}_{0}^{\prime} \cdot \hat{\mathbf{e}}_{0}^{\prime}-\mathbf{e}_{0} \cdot \hat{\mathbf{e}}_{0}\right)+O\left(h^{2}\right)=\frac{1}{2}\left(\left|\mathbf{e}_{0}^{\prime}\right|-\left|\mathbf{e}_{0}\right|\right)+O\left(h^{2}\right) \\
& =\frac{1}{2}\left[\left|\mathbf{e}_{0}+h \sum_{i=1}^{m}\left(\hat{\mathbf{e}}_{i} \times \mathbf{a}_{i+1}+\mathbf{a}_{i} \times \hat{\mathbf{e}}_{i+1}\right)+O\left(h^{2}\right)\right|-\left|\mathbf{e}_{0}\right|\right],
\end{aligned}
$$

which upon expansion through terms of order $h$ yields the expression:

$$
\oiint_{\delta V} d \mathbf{S} \cdot \hat{\mathbf{n}}=\frac{h}{2} \hat{\mathbf{e}}_{0} \cdot \sum_{i=1}^{m}\left(\hat{\mathbf{e}}_{i} \times \mathbf{a}_{i+1}+\mathbf{a}_{i} \times \hat{\mathbf{e}}_{i+1}\right)+O\left(h^{2}\right) .
$$

In writing just this expression and ignoring the contribution from the ends, we have utilized the result

$$
\frac{h}{2} \sum_{i=1}^{m}\left\{\hat{\mathbf{e}}_{i} \cdot\left[\left(\mathbf{a}_{i}-\mathbf{a}_{i+1}\right) \times \hat{\mathbf{e}}_{i}\right]+\hat{\mathbf{e}}_{i+1} \cdot\left[\left(\mathbf{a}_{i}-\mathbf{a}_{i+1}\right) \times \hat{\mathbf{e}}_{i+1}-h\left(\hat{\mathbf{e}}_{i}-\hat{\mathbf{e}}_{i+1}\right) \times \hat{\mathbf{e}}_{i+1}\right]\right\}=0 \text {, }
$$

which shows that the total contribution from the "warped" quadrilateral ends (which have been broken arbitrarily into pairs of triangles) vanishes. We also notice that this particular form of the integral can be rewritten as:

$$
\oiint_{\delta V} d \mathbf{S} \cdot \hat{\mathbf{n}}=\frac{h}{2} \hat{\mathbf{e}}_{0} \cdot \sum_{i=1}^{m}\left[\left(\hat{\mathbf{e}}_{0}+\hat{\mathbf{e}}_{i}+\hat{\mathbf{e}}_{i+1}\right) \times\left(\mathbf{a}_{i+1}-\mathbf{a}_{i}\right)\right]+O\left(h^{2}\right) .
$$


Now let us evaluate the volume of the pillbox. Figure $2 b$ shows that the pillbox is composed of three separate tetrahedrons. Since the volume of

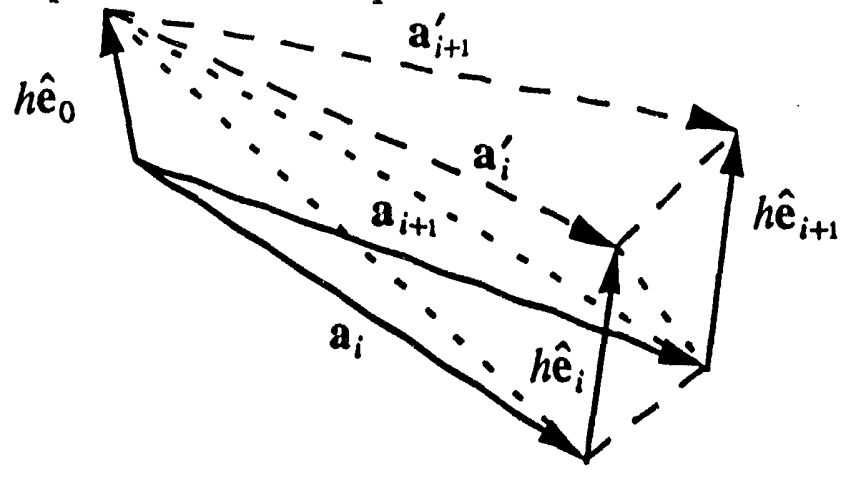

Fig. $2 b$. The pillbox composed of three tetrahedrons.

any tetrahedron of $1 / 6$ of the triple cross produce of the three connected edges at any apex, it follows that the volume can be expressed as

where

$$
V=V_{A}+V_{B}+V_{C} \text {, }
$$

$$
\begin{aligned}
& 6 V_{A}=\sum_{i=1}^{m}\left[h \hat{\mathbf{e}}_{0} \cdot\left(\mathbf{a}_{i} \times \mathbf{a}_{i+1}\right)\right], \\
& 6 V_{B}=\sum_{i=1}^{m}\left\{h \hat{\mathbf{e}}_{i} \cdot\left[\left(\mathbf{a}_{i+1}-\mathbf{a}_{i}\right) \times\left(h \hat{\mathbf{e}}_{i}-\mathbf{a}_{i}\right)\right]\right\},
\end{aligned}
$$

and

$$
6 V_{C}=\sum_{i=1}^{m}\left\{h \hat{\mathbf{e}}_{i+1} \cdot\left[\left(\mathbf{a}_{i}^{\prime}-\mathbf{a}_{i+1}^{\prime}\right) \times \mathbf{a}_{i+1}^{\prime}\right]\right\} .
$$

These expressions may be expanded and combined to yield the expression:

$$
V=\frac{1}{6} h \sum_{i=1}^{m}\left[\left(\hat{\mathbf{e}}_{0}+\hat{\mathbf{e}}_{i}+\hat{\mathbf{e}}_{i+1}\right) \cdot\left(\mathbf{a}_{i} \times \mathbf{a}_{i+1}\right)\right]+O\left(h^{2}\right) .
$$

Consequently, the ratio becomes:

$$
\frac{1}{V} \oiint_{\partial V} d \mathbf{S}=\frac{\hat{\mathbf{e}}_{0} \cdot \sum_{i=1}^{m}\left(\hat{\mathbf{e}}_{i} \times \mathbf{a}_{i+1}+\mathbf{a}_{i} \times \hat{\mathbf{e}}_{i+1}\right)}{\frac{1}{3} \sum_{i=1}^{m}\left[\left(\hat{\mathbf{e}}_{0}+\hat{\mathbf{e}}_{i}+\hat{\mathbf{e}}_{i+1}\right) \cdot\left(\mathbf{a}_{i} \times \mathbf{a}_{i+1}\right)\right]}+O(h) .
$$

Notice that the factors of $h$ cancel, leaving a non zero limit as $h \rightarrow 0$. Several other forms of this expression are useful for interpretation. For example, the numerator can be replaced to give: 


$$
2 J=\frac{\hat{\mathbf{e}}_{0} \cdot \sum_{i=1}^{m}\left[\left(\hat{\mathbf{e}}_{0}+\hat{\mathbf{e}}_{i}+\hat{\mathbf{e}}_{i+1}\right) \times\left(\mathbf{a}_{i+1}-\mathbf{a}_{i}\right)\right]}{\frac{1}{3} \sum_{i=1}^{m}\left[\left(\hat{\mathbf{e}}_{0}+\hat{\mathbf{e}}_{i}+\hat{\mathbf{c}}_{i+1}\right) \cdot\left(\mathbf{a}_{i} \times \mathbf{a}_{i+1}\right)\right]} .
$$

Alternatively, both numerator and denominator can be rewritten so as to involve the differences, $\hat{\mathbf{e}}_{i}-\hat{\mathbf{e}}_{0}$ and $\hat{\mathbf{e}}_{i+1}-\hat{\mathbf{e}}_{0}$. Then we obtain

$$
2 J=\frac{\hat{\mathbf{e}}_{0} \cdot \sum_{i=1}^{m}\left[\left(\hat{\mathbf{e}}_{i}-\hat{\mathbf{e}}_{0}\right) \times \mathbf{a}_{i+1}+\mathbf{a}_{i} \times\left(\hat{\mathbf{e}}_{i+1}-\hat{\mathbf{e}}_{0}\right)\right]}{\hat{\mathbf{e}}_{0} \cdot \sum_{i=1}^{m}\left(\mathbf{a}_{i} \times \mathbf{a}_{i+1}\right)+\frac{1}{3} \sum_{i=1}^{m}\left\{\left[\left(\hat{\mathbf{e}}_{i}-\hat{\mathbf{e}}_{0}\right)+\left(\hat{\mathbf{e}}_{i+1}-\hat{\mathbf{e}}_{0}\right)\right] \cdot\left(\mathbf{a}_{i} \times \mathbf{a}_{i+1}\right)\right\}} .
$$

It is of interest to demonstrate that these expressions yield the correct result for some special cases. For example, if all the vectors $\hat{\mathbf{e}}_{i}$ are parallel to $\hat{\mathbf{e}}_{0}$ then their differences vanish, so $J$ vanishes consistent with the planar character of this portion of the surface. Next consider the special case of a sphere on which all the vectors, $a_{i}$, have the same magnitude. In this particular case, we already have shown that the unit vectors, $\hat{\mathbf{e}}_{i}$, coincide with radial unit vectors and hence, that $R\left(\hat{\mathbf{e}}_{i}-\hat{\mathbf{e}}_{0}\right)=\mathbf{a}_{i}$. Therefore, the numerator in the above expression becomes

$$
\frac{1}{R} \hat{\mathbf{e}}_{0} \cdot \sum_{i=1}^{m}\left[R\left(\hat{\mathbf{e}}_{i}-\hat{\mathbf{e}}_{0}\right) \times \mathbf{a}_{i+1}+\mathbf{a}_{i} \times R\left(\hat{\mathbf{e}}_{i+1}-\hat{\mathbf{e}}_{0}\right)\right]=\frac{2}{R} \hat{\mathbf{e}}_{0} \cdot \sum_{i=1}^{m} \mathbf{a}_{i} \times \mathbf{a}_{i+1},
$$

and the second term in the denominator vanishes, so the ratio yields the value, $2 / R$, in agreement with expectations.

Let us digress briefly to analyze the structure of the formula:

$$
2 J=\frac{\hat{\mathbf{e}}_{0} \cdot \frac{1}{2} \sum_{i=1}^{m}\left[\left(\hat{\mathbf{e}}_{0}+\hat{\mathbf{e}}_{i}+\hat{\mathbf{e}}_{i+1}\right) \times\left(\mathbf{a}_{i+1}-\mathbf{a}_{i}\right)\right]}{\sum_{i=1}^{m}\left[\frac{1}{3}\left(\hat{\mathbf{e}}_{0}+\hat{\mathbf{e}}_{i}+\hat{\mathbf{e}}_{i+1}\right) \cdot \frac{1}{2}\left(\mathbf{a}_{i} \times \mathbf{a}_{i+1}\right)\right]} .
$$

The numerator represents a sum of approximations to line integrals around each of the triangular elements which surround the central node. Consider the sum:

$$
\left(\hat{\mathbf{e}}_{i}+\hat{\mathbf{e}}_{i+1}\right) \times\left(\mathbf{a}_{i+1}-\mathbf{a}_{i}\right)+\left(\hat{\mathbf{e}}_{i+1}+\hat{\mathbf{e}}_{0}\right) \times\left(-\mathbf{a}_{i+1}\right)+\left(\hat{\mathbf{e}}_{0}+\hat{\mathbf{e}}_{i}\right) \times \mathbf{a}_{i},
$$

which consists of an average "normal" vector crossed into the associated line segment separating the two normals and directed in the "positive" direction. This expression can be rearranged and summed over all the elements surrounding the central node. Only the contribution from the first 
term survives, and this is precisely summation in the numerator of the above formula because the summation over $\hat{\mathbf{e}}_{0} \times\left(\mathbf{a}_{i+1}-\mathbf{a}_{i}\right)$ makes no contribution. In the same spirit, the denominator appears to represent the projection of the area of each triangular element in the direction of an average normal, $\frac{1}{3}\left(\hat{\mathbf{e}}_{0}+\hat{\mathbf{e}}_{i}+\hat{\mathbf{e}}_{i+1}\right)$, associated with that surface element.

All this suggests that there must exist an alternative expression for the mean curvature of a surface. By applying Stokes' circulation theorem:

$$
\iint_{S}(\nabla \times \mathbf{A}) \cdot d \mathbf{S}=\oint_{C} \mathbf{A} \cdot d \mathbf{r}
$$

to the vector cross product, $\mathbf{c} \times \mathbf{B}$, where $\mathbf{c}$ is an arbitrary constant vector, one can obtain the alternative form:

$$
\iint_{S}(\hat{\mathbf{n}} \times \nabla) \times \mathbf{B} d \mathbf{S}=\oint_{C} d \mathbf{r} \times \mathbf{B} .
$$

If the arbitrary vector, $\mathbf{B}$, is chosen to be the unit normal, then ${ }^{3}$ after some manipulation it follows that

$$
2 J \hat{\mathbf{n}}=\lim _{S \rightarrow 0} \frac{1}{S} \oint_{C} \hat{\mathbf{n}} \times d \mathbf{r}
$$

where the integration is taken in the positive direction along the simple closed curve, $C$, which bounds an open, two-sided surface with area, $S$. This formula clearly shows that the first or mean curvature of a surface is a heuristic measure 4 of the turn of the normal direction for a unit step along the surface averaged over all directions.

Our formula for the mean curvature can be recast into an even closer correspondence with this general expression by "factoring" out the vector, $\hat{\mathbf{e}}_{0}$ so as to obtain

$$
2 J \hat{\mathbf{e}}_{0}=\frac{\sum_{i=1}^{m}\left[\frac{1}{2}\left(\hat{\mathbf{e}}_{i}+\hat{\mathbf{e}}_{i+1}\right) \times\left(\mathbf{a}_{i+1}-\mathbf{a}_{i}\right)\right]}{\sum_{i=1}^{m}\left[\frac{1}{3}\left(\hat{\mathbf{e}}_{0}+\hat{\mathbf{e}}_{i}+\hat{\mathbf{e}}_{i+1}\right) \cdot \frac{1}{2}\left(\mathbf{a}_{i} \times \mathbf{a}_{i+1}\right)\right]},
$$

in which it is to be noted that the right-hand side represents a discrete approximation of the line integral divided by a discrete approximation for the enclosed area (prior to taking any limit). Moreover, suggestive factors of $1 / 2$ have been inserted to identify just exactly where the interpretation of this ratio as a mean or average quantity actually is manifest. 
Thus, we argue that the first (or mean) curvature of the surface is represented by these expressions. There are several points to notice about these formulas. First, we observe that these expressions depend not only upon the approximate normal assigned at the central node, labeled by 0 , but also depend upon the normals assigned to the "ring" of neighboring nodes, labeled by $i=1,2, \ldots, m$, and these normals depend upon the locations of still further "next nearest" neighboring nodes. Next we note that if the neighboring normals are parallel to the central normal, then all their cross products vanish and the curvature vanishes at the central node. Finally, we observe that this particular expression is constructed directly from the geometrical orientation of the edges of the finite elements and the locations of their corners (referred to here as nodes) and does not involve interpolation functions commonly employed in finite element analysis.

\section{Triangular Elements}

Heretofore, we have considered a construction scheme which is based solely upon the nodes. Each node resides on the actual surface and all of the constructed vectors, which represent normal directions, are tied to (emanate from) nodal points. All of the nodes surrounding the central node were utilized as corners of associated triangular patches which always had one vertex at the central node. However, there was no explicit requirement that the edge opposite the vertex had to be the edge of a finite element. Now let us contemplate a situation in which all of the elements are triangular. Clearly, the foregoing construction can be use to assign a unique unit vector to each surface node. Is this sufficient, or does one gain some simplification by also utilizing the unit normal associated with the triangular element itself? Clearly, the nodes of each triangular element must be coplanar, so the vector cross- products formed from any pair of edges will be parallel (and equal) with a magnitude equal to twice the area of the enclosed triangle. This means that

$$
a_{1} \times a_{2}=a_{2} \times a_{3}=a_{3} \times a_{1}=\frac{1}{3} e_{0}=\frac{1}{3} \sum_{i=1}^{3} a_{i} \times a_{i+1},
$$

and from this a unit vector, $\hat{\mathbf{e}}_{0}$, can be constructed in the usual fashion. This unit vector has the same direction at every point within the triangle, so it should not matter at what point we choose to place it. In order to investigate this conjecture, we shall construct a new set of vectors, $\left\{\mathbf{b}_{1}, \mathbf{b}_{2}, \mathbf{b}_{3}\right\}$, which meet at a common point within the triangle and when extended divide with a given ratio the side opposite each vertex. The geometrical configuration is illustrated in Fig. 3. Each of these sets can be re-expressed in terms of the other. For example, 


$$
a_{1}=b_{1}-b_{2} \text {, etc. and }\left(1+m_{1}\right) b_{1}=\left(1-f_{2}\right) a_{1}-f_{2} a_{3} \text {, etc., }
$$

where "etc." means "complete the set of three by cyclic permutation." The edges are divided by the fractions, $f_{i}$, and the internal extensions are

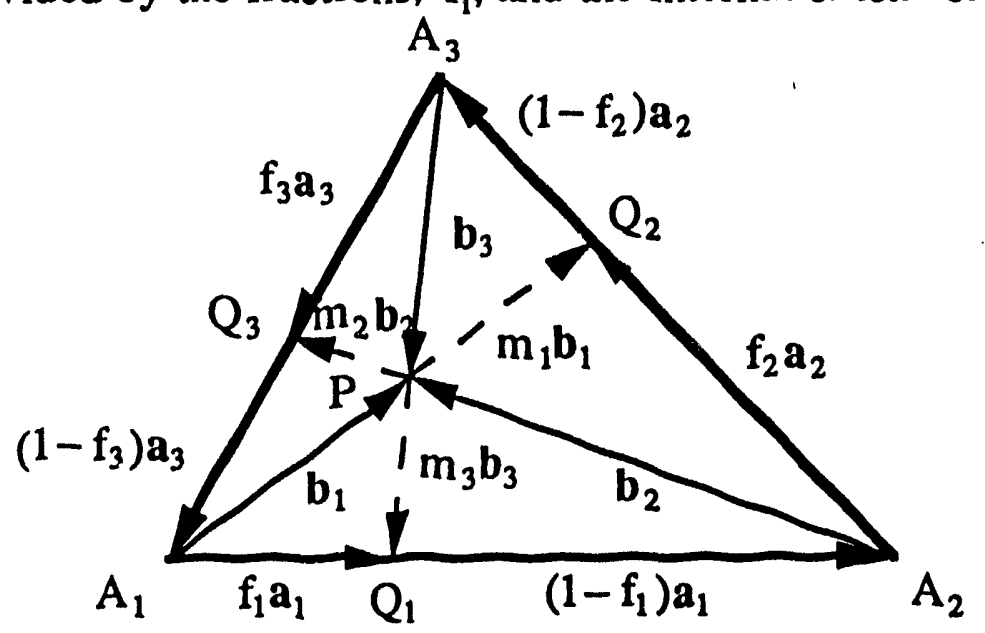

Fig. 3. Arbitrary location of normal within a triangular element.

increased by the fractions, $m_{i}$. When all these fractions are equal to $1 / 2$, the b-type vectors coincide with the medians of the triangle and meet at the common point, $P$, which is the center of gravity. A variant of the theorem of Ceva, 5,6 specifies certain relationships which these fractions must satisfy under these conditions. Since none of the six basic vectors in Fig. 3 are collinear, it follows that these factors must satisfy three basic sets of relationships:

and

$$
f_{1}\left(1+m_{1}\right)=\left(1+m_{3}\right)\left(1-f_{2}\right) \text {, etc., } f_{1}\left(1+m_{2}\right)=m_{2}\left(1+m_{3}\right) \text {, etc., }
$$

By combining these it also follows that:

$$
f_{1} f_{2} f_{3}=\left(1-f_{1}\right)\left(1-f_{2}\right)\left(1-f_{3}\right)=m_{1} m_{2} m_{3} \text {. }
$$

(A large number of more complicated expressions also can be derived from these relationships.)

We are interested in the combination:

$$
\begin{aligned}
\sum_{i=1}^{3} \mathbf{a}_{i} \times \mathbf{a}_{i+1} & =\sum_{i=1}^{3}\left(\mathbf{b}_{i}-\mathbf{b}_{i+1}\right) \times\left(\mathbf{b}_{i+1}-\mathbf{b}_{i+2}\right) \\
& =\sum_{i=1}^{3}\left[\left(\mathbf{b}_{i} \times \mathbf{b}_{i+1}\right)+\left(\mathbf{b}_{i+1} \times \mathbf{b}_{i+2}\right)+\left(\mathbf{b}_{i+2} \times \mathbf{b}_{i}\right)\right] \\
& =3 \sum_{i=1}^{3}\left(\mathbf{b}_{i} \times \mathbf{b}_{i+1}\right),
\end{aligned}
$$


which shows that the same unit normal, $\hat{\mathbf{e}}_{0}$, will be obtained at any arbitrary point $P$ regardless of which set of vectors is utilized. A geometric proof also is self-evident because all the cross-products are parallel and each has a magnitude equal to twice the area of the associated triangle. Since the sum of the cross-products of the b's covers the total area twice while each cross-product of the a's covers the whole triangle twice, the sum of all three terms will cover the triangle a total of six times, thus giving the anticipated three-to-one ratio between the two sums.

Now let us examine how to associate a mean curvature to the surface in the neighborhood of this particular point. We have at our disposal an averaged unit normal, $\hat{\mathbf{e}}_{0}$, at a particular point within the triangle, and by making use of the previously described construction for triangular patches surrounding each nodal point, we also can associate a unique unit normal, $\left\{\hat{\mathbf{e}}_{i} \mid i=1,2,3\right\}$, with each of the vertices of this element. (Notice that these unit vectors are not parallel to $\hat{\mathbf{e}}_{0}$.) These unit vectors may be used to construct a pillbox by displacing the element a uniform distance $h$ along each of the unit vectors. The geometrical configuration is similar to that illustrated in Fig. 2a. except for the labeling of the vectors, $a_{i}$,

Now let us proceed to form the surface integral over the faces of this pillbox. For the sake of symmetry it is convenient to utilize the internal vectors, $\mathbf{b}_{i}$, rather than the edges, $\mathbf{a}_{i}$. The desired integral over the base has the form:

$$
\begin{aligned}
\iint_{\text {base }} d \mathbf{S} \cdot \hat{\mathbf{e}}_{0} & \approx \sum_{i=1}^{3} \Delta \mathbf{S}_{i} \cdot \hat{\mathbf{e}}_{0} \approx \hat{\mathbf{e}}_{0} \cdot \sum_{i=1}^{3} \hat{\mathbf{n}}_{i} \Delta \mathbf{S} \approx \hat{\mathbf{e}}_{0} \cdot \sum_{i=1}^{3} \frac{\mathbf{b}_{i} \times \mathbf{b}_{i+1}}{\left|\mathbf{b}_{i} \times \mathbf{b}_{i+1}\right|} \frac{1}{2}\left|\mathbf{b}_{i} \times \mathbf{b}_{i+1}\right| \\
& =\frac{1}{2} \hat{\mathbf{e}}_{0} \cdot \sum_{i=1}^{3} \mathbf{b}_{i} \times \mathbf{b}_{i+1}=\frac{1}{6} \hat{\mathbf{e}}_{0} \cdot \sum_{i=1}^{3} \mathbf{a}_{i} \times \mathbf{a}_{i+1}=\frac{1}{6} \hat{\mathbf{e}}_{0} \cdot \mathbf{e}_{0}=\frac{1}{6}\left|\mathbf{e}_{0}\right| .
\end{aligned}
$$

Apart from a sign and an extra factor of $1 / 3$, which arises because of the 3:1 ratio between areas computed with the a's instead of the b's. The integral over the top of the pillbox can be constructed similarly. The result, ignoring contributions from the sides which are of order $h^{2}$, is

$$
\oiint_{\delta V} d \mathbf{S} \cdot \hat{\mathbf{n}}=\iint_{t o p} d \mathbf{S}^{\prime} \cdot \hat{\mathbf{e}}_{0}^{\prime}+\iint_{\text {base }} d \mathbf{S} \cdot \hat{\mathbf{e}}_{0}+O\left(h^{2}\right) \approx \frac{1}{6}\left(\mathbf{e}_{0}^{\prime} \cdot \hat{\mathbf{e}}_{0}^{\prime}-\mathbf{e}_{0} \cdot \hat{\mathbf{e}}_{0}\right)+O\left(h^{2}\right) .
$$

The negative sign arises because the integral utilizes the outward-drawn normal which gives a negative contribution over the base because $\hat{\mathbf{e}}_{0}$ points into rather than out from the volume. This expression may be expanded in exactly the same fashion as before by making use of the expansion 


$$
\begin{aligned}
\mathbf{e}_{0}^{\prime} & =3 \sum_{i=1}^{3} \mathbf{b}_{i}^{\prime} \times \mathbf{b}_{i+1}^{\prime}=3 \sum_{i=1}^{3}\left\{\left[\mathbf{b}_{i}-h\left(\hat{\mathbf{e}}_{i}-\hat{\mathbf{e}}_{0}\right)\right] \times\left[\mathbf{b}_{i+1}-h\left(\hat{\mathbf{e}}_{i+1}-\hat{\mathbf{e}}_{0}\right)\right]\right\} \\
& \left.=3 \sum_{i=1}^{3}\left\{\mathbf{b}_{i} \times \mathbf{b}_{i+1}-h\left[\hat{\mathbf{e}}_{i} \times \mathbf{b}_{i+1}+\mathbf{b}_{i} \times \hat{\mathbf{e}}_{i+1}+\hat{\mathbf{e}}_{0} \times\left(\mathbf{b}_{i}-\mathbf{b}_{i+1}\right)\right]+O\left(h^{2}\right)\right)\right\} \\
& =\mathbf{e}_{0}+3 h \sum_{i=1}^{3}\left[\left(\hat{\mathbf{e}}_{0}+\hat{\mathbf{e}}_{i}+\hat{\mathbf{e}}_{i+1}\right) \times\left(\mathbf{b}_{i}-\mathbf{b}_{i+1}\right)\right]+O\left(h^{2}\right) \\
& =\mathbf{e}_{0}+3 h \sum_{i=1}^{3}\left[\left(\hat{\mathbf{e}}_{0}+\hat{\mathbf{e}}_{i}+\hat{\mathbf{e}}_{i+1}\right) \times \mathbf{a}_{i}\right]+O\left(h^{2}\right) .
\end{aligned}
$$

When this expression is substituted into the integral and expanded to order $h$, we obtain the result:

$$
\oiint_{\delta V} d \mathbf{S} \cdot \hat{\mathbf{n}}=\frac{h}{2} \hat{\mathbf{e}}_{0} \cdot \sum_{i=1}^{3}\left[\left(\hat{\mathbf{e}}_{0}+\hat{\mathbf{e}}_{i}+\hat{\mathbf{e}}_{i+1}\right) \times \mathbf{a}_{i}\right]+O\left(h^{2}\right) .
$$

Similarly, let us compute the volume of the enclosed figure. It can be partitioned into three tetrahedrons as before so

where

$$
V=V_{A}+V_{B}+V_{C}
$$

$$
\begin{aligned}
& 6 V_{A}=h \hat{\mathbf{e}}_{1} \cdot\left[\mathbf{a}_{1} \times\left(-\mathbf{a}_{3}\right)\right], \\
& 6 V_{B}=h \hat{\mathbf{e}}_{2} \cdot\left[\mathbf{a}_{2} \times\left(h \hat{\mathbf{e}}_{1}-\mathbf{a}_{1}\right)\right],
\end{aligned}
$$

and

$$
6 V_{c}=h \hat{\mathbf{e}}_{2} \cdot\left[\mathbf{a}_{3}^{\prime} \times\left(-\mathbf{a}_{2}^{\prime}\right)\right] \text {. }
$$

These expressions may be expanded and combined to yield the expression:

$$
\begin{aligned}
6 V & =h\left[\hat{\mathbf{e}}_{1} \cdot\left(\mathbf{a}_{3} \times \mathbf{a}_{1}\right)+\hat{\mathbf{e}}_{2} \cdot\left(\mathbf{a}_{1} \times \mathbf{a}_{2}\right)+\hat{\mathbf{e}}_{3} \cdot\left(\mathbf{a}_{2} \times \mathbf{a}_{3}\right)\right]+O\left(h^{2}\right) \\
& =h\left(\hat{\mathbf{e}}_{1}+\hat{\mathbf{e}}_{2}+\hat{\mathbf{e}}_{3}\right) \cdot\left(\mathbf{a}_{3} \times \mathbf{a}_{1}\right)=\frac{1}{3} h\left(\hat{\mathbf{e}}_{1}+\hat{\mathbf{e}}_{2}+\hat{\mathbf{e}}_{3}\right) \cdot \sum_{i=1}^{3}\left(\mathbf{a}_{i} \times \mathbf{a}_{i+1}\right)+O\left(h^{2}\right) \\
& =\frac{1}{3} h\left(\hat{\mathbf{e}}_{1}+\hat{\mathbf{e}}_{2}+\hat{\mathbf{e}}_{3}\right) \cdot \mathbf{e}_{0}+O\left(h^{2}\right) .
\end{aligned}
$$

In this manner the b-type vectors are eliminated completely from the final expressions. Consequently, in limit as $h \rightarrow 0$ the ratio becomes:

$$
2 J=\frac{\frac{1}{2} \hat{\mathbf{e}}_{0} \cdot \sum_{i=1}^{3}\left[\left(\hat{\mathbf{e}}_{i}+\hat{\mathbf{e}}_{i+1}\right) \times \mathbf{a}_{i}\right]}{\frac{1}{6}\left[\hat{\mathbf{e}}_{1} \cdot\left(\mathbf{a}_{3} \times \mathbf{a}_{1}\right)+\hat{\mathbf{e}}_{2} \cdot\left(\mathbf{a}_{1} \times \mathbf{a}_{2}\right)+\hat{\mathbf{e}}_{3} \cdot\left(\mathbf{a}_{2} \times \mathbf{a}_{3}\right)\right]}
$$

and by "factoring" out the vector, $\hat{\mathbf{e}}_{0}$, from the numerator, one alternatively gets the form: 


$$
2 J \hat{\mathbf{e}}_{0}=\frac{\sum_{i=1}^{3}\left[\left(\hat{\mathbf{e}}_{i}+\hat{\mathbf{e}}_{i+1}\right) \times \mathbf{a}_{i}\right]}{\frac{1}{9}\left(\hat{\mathbf{e}}_{1}+\hat{\mathbf{e}}_{2}+\hat{\mathbf{e}}_{3}\right) \cdot \mathbf{e}_{0}}=\frac{\sum_{i=1}^{3}\left[\frac{1}{2}\left(\hat{\mathbf{e}}_{i}+\hat{\mathbf{e}}_{i+1}\right) \times \mathbf{a}_{i}\right]}{\left[\frac{1}{3}\left(\hat{\mathbf{e}}_{1}+\hat{\mathbf{e}}_{2}+\hat{\mathbf{e}}_{3}\right) \cdot \frac{1}{6} \mathbf{e}_{0}\right]} .
$$

This form again represents the ratio of an approximation for the line integral divided by an approximation to the area enclosed. (Observe that $\frac{1}{6} \mathbf{e}_{0}$ actually is the area of the triangle.) The second form, in which extra factors of $1 / 2$ have been distributed, more clearly reflects the interpretation of $J$ as a mean or average curvature.

\section{Quadrilateral Elements}

Heretofore, we have considered a construction scheme which is based solely upon the nodes. Each node resides on the actual surface and all of the constructed vectors, which represent normal directions, are tied to (emanate from) nodal points. All of the nodes surrounding the central node were utilized as corners of associated triangular patches which always had one vertex at the central node. (However, there was no explicit requirement that the edge opposite the vertex had to be the edge of a finite element.) Now we want to examine schemes that might be applicable to warped quadrilateral elements. Even though each of the four nodes resides on the curved surface, this type of element will be warped because its sides will not be coplanar in general, and moreover, the pair of diagonals need not even touch each other. The general situation is illustrated in Fig. 4a. The corners are numbered successively counterclockwise and each side is assigned a vector, $\mathbf{a}_{i}$.

There are at least two issues to resolve. First, it would be desirable to assign to this element a unique unit vector, which suitably represents the direction normal to the enclosed area. Second, it would be desirable to specify where, that is to say at what point, on the actual surface within the apparent confines of the enclosed area this normal vector is to be located. Unfortunately, neither of these desires can be satisfied easily. The element may be warped, and therefore not possess the same normal direction at every point within the enclosed area. Moreover, no information is given about the elevation of the actual surface within this projected area, so the desired location cannot be found casily. However, we can construct a set of four new vectors: $\left\{\mathbf{a}_{i} \times \mathbf{a}_{i+1} \mid i=1,2,3,4,5=1 \bmod (4)\right\}$ from the cross products of successive pairs of these vectors. These vectors are perpendicular to the pair of edges which were used in their construction, but are not necessarily parallel. We assert that all of them should be used 


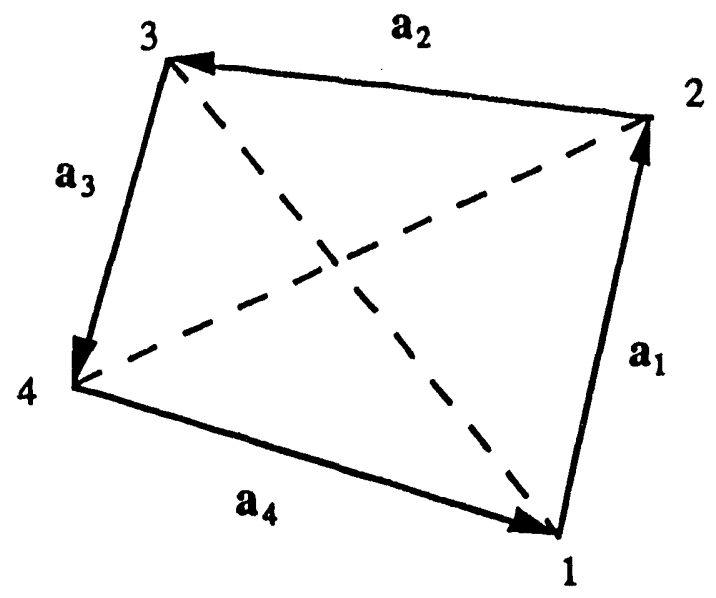

Fig. 4a. Basic warped quadrilateral element.

in the construction of a normal to be associated with this element. There are several ways by which this assignment may be accomplished.

One of the simplest ways to construct a composite vector from this set is to form the sum:

$$
\mathbf{e}_{0}=\sum_{i=1}^{4} \mathbf{a}_{i} \times \mathbf{a}_{i+1} \text {, and the associated unit vector, } \hat{\mathbf{e}}_{0}=\frac{\mathbf{e}_{0}}{\left|\mathbf{e}_{0}\right|} .
$$

This represents a unitary arithmetic average of the set of four vectors. Alternatively, one could have first normalized each of the four vectors in the set to unity, and then formed the sum to obtain a kind of arithmetic average of unit vectors. The magnitude of this sum would have the value 4 , which is just the sum of the individual magnitudes, if all the unit vectors happened to be parallel, but otherwise would be a more complicated normalization factor. However, in view of our earlier analysis of triangular patches, it would seem appropriate to also take into account some associated area. Hence, let us form the summation:

$$
\sum_{i=1}^{4}\left[\left(\frac{\mathbf{a}_{i} \times \mathbf{a}_{i+1}}{\left|\mathbf{a}_{i} \times \mathbf{a}_{i+1}\right|}\right) \frac{1}{2}\left|\mathbf{a}_{i} \times \mathbf{a}_{i+1}\right|\right]=\frac{1}{2} \mathbf{e}_{0},
$$

whose terms consist of the individual unit vector multiplied by the area of the associated triangle. It is easily seen that this sum is exactly half of the vector, $e_{0}$, which was defined initially. Since the factor of $1 / 2$ will disappear upon normalization, there is no loss of generality in omitting it altogether. Hence, it would seem appropriate to proceed with our analysis using the vector, $\mathbf{e}_{0}$, for the normal direction associated with this warped quadrilateral element. 
There is another method of construction by which a normal vector can be associated with this quadrilateral. Let us form the vector cross product using the pair of principal diagonals, $\mathbf{a}_{1}+\mathbf{a}_{2}$ and $\mathbf{a}_{2}+\mathbf{a}_{3}$, and equivalently, the pair, $-\left(a_{3}+a_{4}\right)$ and $-\left(a_{4}+a_{1}\right)$. We obtain the vector:

$$
\begin{aligned}
n & =\left(a_{1}+a_{2}\right) \times\left(a_{2}+a_{3}\right)=a_{1} \times a_{2}+a_{2} \times a_{3}+a_{1} \times a_{3} \\
& =-\left(a_{3}+a_{4}\right) \times\left[-\left(a_{4}+a_{1}\right)\right]=a_{3} \times a_{4}+a_{4} \times a_{1}-a_{1} \times a_{3} \\
& =\frac{1}{2}\left(a_{1} \times a_{2}+a_{2} \times a_{3}+a_{3} \times a_{4}+a_{4} \times a_{1}\right)=\frac{1}{2} e_{0} .
\end{aligned}
$$

So, this alternative construction method also generates the same vector, $\frac{1}{2} \mathbf{e}_{0}$. It is easy to show that if all four vertices of the quadrilateral are coplanar, then the enclosed area is given by the magnitude of $\frac{1}{4} e_{0}$, and if the vertices are not coplanar (i.e., the quadrilateral is warped), then $\frac{1}{4}\left|e_{0}\right|$ is the area projected onto a plane perpendicular to $\hat{\mathbf{e}}_{0}$.

Now let us assign the origin of the vector, $\hat{\mathbf{e}}_{0}$, to the midpoint of the line connecting the midpoints of the principal diagonals, $a_{1}+a_{2}$ and $a_{2}+a_{3}$, of the quadrilateral. If the vector, $2 x$, connects the midpoints of the principal diagonals as in Fig. $4 \mathrm{~b}$, then

$$
a_{1}+\frac{1}{2}\left(a_{2}+a_{3}\right)-2 x-\frac{1}{2}\left(a_{1}+a_{2}\right)=0 \Rightarrow x=\frac{1}{4}\left(a_{1}+a_{3}\right) \text {, }
$$

and because

$$
a_{1}+a_{2}+a_{3}+a_{4}=0 \Rightarrow x=\frac{1}{8}\left(a_{1}-a_{2}+a_{3}-a_{4}\right) \text {. }
$$

The vector from the $i$ th node to this central point is

$$
\mathbf{b}_{i}=\frac{1}{4}\left(3 \mathbf{a}_{i}+2 \mathbf{a}_{i+1}+\mathbf{a}_{i+2}\right)=\frac{1}{8}\left(3 \mathbf{a}_{i}+\mathbf{a}_{i+1}-\mathbf{a}_{i+2}-3 \mathbf{a}_{i+3}\right) \text {. }
$$

There are four vectors, $\left\{\mathbf{b}_{1}, \mathbf{b}_{2}, \mathbf{b}_{3}, \mathbf{b}_{4}\right\}$, in this new set. Successive vector cross products have the property that:

$$
\begin{aligned}
\mathbf{b}_{i} \times \mathbf{b}_{i+1} & =\left(\mathbf{b}_{i}-\mathbf{b}_{i+1}\right) \times \mathbf{b}_{i+1}=-\mathbf{b}_{i} \times\left(\mathbf{b}_{i}-\mathbf{b}_{i+1}\right) \\
& =\frac{1}{2}\left(\mathbf{b}_{i}-\mathbf{b}_{i+1}\right) \times\left(\mathbf{b}_{i}+\mathbf{b}_{i+1}\right) \\
& =\frac{1}{2} \mathbf{a}_{i} \times \frac{1}{4}\left(2 \mathbf{a}_{i}+4 \mathbf{a}_{i+1}\right)=\frac{1}{2} \mathbf{a}_{i} \times \mathbf{a}_{i+1},
\end{aligned}
$$

so the cyclic sum of successive cross products satisfies the relationship:

$$
\sum_{i=1}^{4} \mathbf{b}_{i} \times \mathbf{b}_{i+1}=\frac{1}{2} \sum_{i=1}^{4} \mathbf{a}_{i} \times \mathbf{a}_{i+1}=\frac{1}{2} \mathbf{e}_{0} .
$$

It follows from this result that in so far as the construction of the normal direction is concerned, this new set of vectors, $\left\{\mathbf{b}_{1}, \mathbf{b}_{2}, \mathbf{b}_{3}, \mathbf{b}_{4}\right\}$, can be used as an equivalent set for construction of the unit normal, $\hat{\mathbf{e}}_{0}$, in place of the original set of vectors, $\left\{\mathbf{a}_{1}, \mathbf{a}_{2}, \mathbf{a}_{3}, \mathbf{a}_{4}\right\}$, which connect the actual surface nodes. Each of the vectors in this new set points from a surface node to the designated origin of the unit normal vector, $\hat{\mathbf{e}}_{0}$, as shown in Fig. $4 \mathrm{~b}$. 
These vectors are not necessarily coplanar, nor is their common endpoint located on the actual surface. Nevertheless, this construction generates a unique unit vector at a specific location for each quadrilateral element.

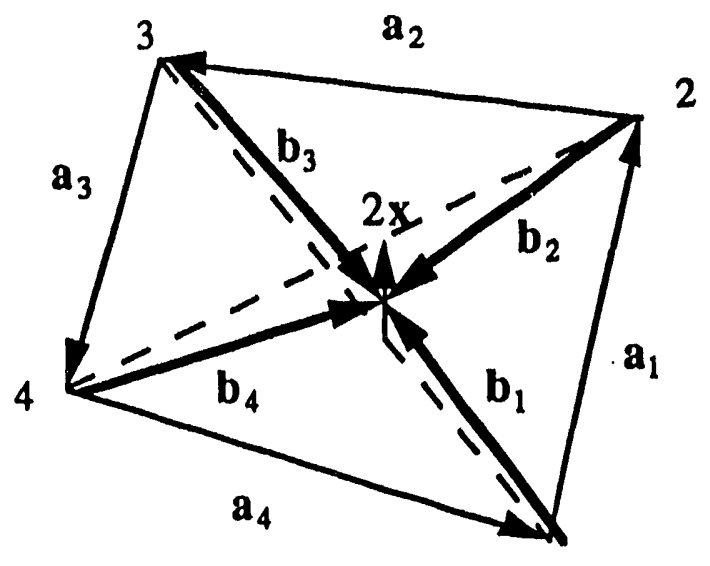

Fi ;. 4b. New set of basis vectors directed toward the position of the unit normal.

Now let us examine how to associate a mean curvature to the surface in the neighborhood of this particular point. We have at our disposal an averaged unit normal, $\hat{\mathbf{e}}_{0}$, at a particular point within the quadrilateral, and by making use of the previously described construction for triangular patches surrounding each nodal point, we also can associate a unique unit normal, $\left\{\dot{e}_{i} \mid i=1,2,3,4\right\}$, with each of the four nodal points of this element. These unit vectors may be used to construct a quadrilateral-like pillbox by displacing the warped quadrilateral a uniform distance $h$ along each of the unit vectors. Let us choose for the area to be associated with the base, not that of the original quadrilateral, but the roof-like area consisting of the four triangles formed by the vectors, $\mathbf{b}_{i}$. Similarly, we shall utilize the perturbed vectors, $b_{i}^{\prime}$, to form another roof-like area for the top of the figure. All this is illustrated in Fig. 5.

Now form the surface integral over the faces of this pillbox. The desired integral over the base has the form:

$$
\begin{aligned}
\iint_{\text {base }} d \mathbf{S} \cdot \hat{\mathbf{e}}_{0} & \approx \sum_{i=1}^{4} \Delta \mathbf{S}_{i} \cdot \hat{\mathbf{e}}_{0} \approx \hat{\mathbf{e}}_{0} \cdot \sum_{i=1}^{4} \hat{\mathbf{n}}_{i} \Delta \mathbf{S} \approx \hat{\mathbf{e}}_{0} \cdot \sum_{i=1}^{4} \frac{\mathbf{b}_{i} \times \mathbf{b}_{i+1}}{\left|\mathbf{b}_{i} \times \mathbf{b}_{i+1}\right|} \frac{1}{2}\left|\mathbf{b}_{i} \times \mathbf{b}_{i+1}\right| \\
& =\frac{1}{2} \hat{\mathbf{e}}_{0} \cdot \sum_{i=1}^{4} \mathbf{b}_{i} \times \mathbf{b}_{i+1}=\frac{1}{4} \hat{\mathbf{e}}_{0} \cdot \sum_{i=1}^{4} \mathbf{a}_{i} \times \mathbf{a}_{i+1}=\frac{1}{4} \hat{\mathbf{e}}_{0} \cdot \mathbf{e}_{0}=\frac{1}{4}\left|\mathbf{e}_{0}\right| .
\end{aligned}
$$




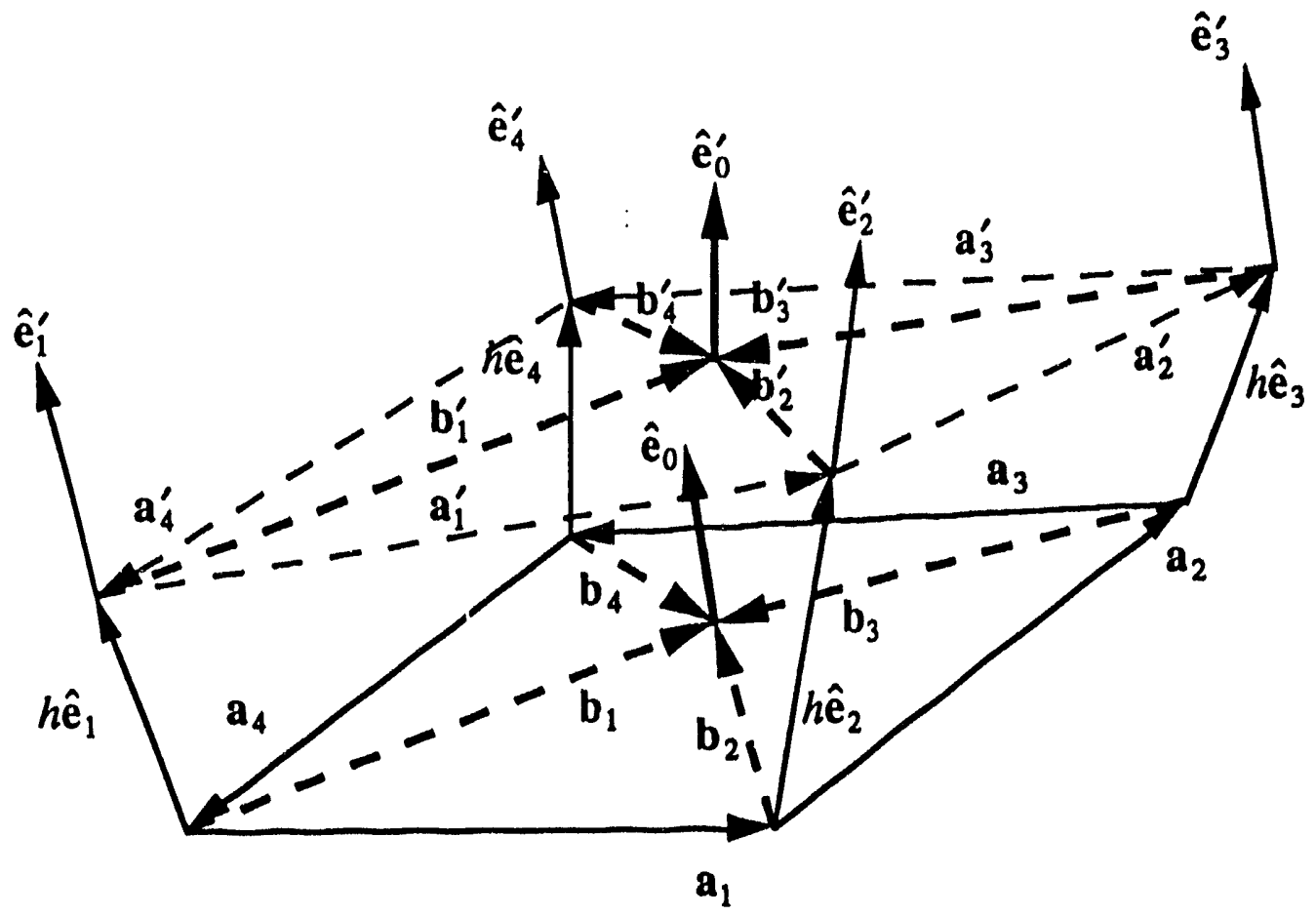

Fig. 5. The "quadrilateral-like" pillbox.

Apart from a sign and an extra factor of $1 / 2$, which arises because the area computed with the vectors $a_{i}$ accounts for the area of the quadrilateral twice, this is the same result that was obtained earlier in the case of the triangular patches. Similarly, the integral over the top of the pillbox can be constructed. The result, ignoring contributions from the sides which are of order $h^{2}$, is

$$
\oiint_{\delta V} d \mathbf{S} \cdot \hat{\mathbf{n}}=\iint_{\text {lop }} d \mathbf{S}^{\prime} \cdot \hat{\mathbf{e}}_{0}^{\prime}+\iint_{\text {base }} d \mathbf{S} \cdot \hat{\mathbf{e}}_{0}+O\left(h^{2}\right) \approx \frac{1}{4}\left(\mathbf{e}_{0}^{\prime} \cdot \hat{\mathbf{e}}_{0}^{\prime}-\mathbf{e}_{0} \cdot \hat{\mathbf{e}}_{0}\right)+O\left(h^{2}\right) .
$$

The negative sign arises because the integral utilizes the outward-drawn normal which gives a negative contribution over the base because $\hat{\mathbf{e}}_{0}$ points into rather than out from the volume. This expression may be expanded in exactly the same fashion as before by making use of the expansion

$$
\begin{aligned}
\mathbf{e}_{0}^{\prime} & =2 \sum_{i=1}^{4} \mathbf{b}_{i}^{\prime} \times \mathbf{b}_{i+1}^{\prime}=2 \sum_{i=1}^{4}\left\{\left[\mathbf{b}_{i}-h\left(\hat{\mathbf{e}}_{i}-\hat{\mathbf{e}}_{0}\right)\right] \times\left[\mathbf{b}_{i+1}-h\left(\hat{\mathbf{e}}_{i+1}-\hat{\mathbf{e}}_{0}\right)\right]\right\} \\
& =2 \sum_{i=1}^{4}\left\{\mathbf{b}_{i} \times \mathbf{b}_{i+1}-h\left[\hat{\mathbf{e}}_{i} \times \mathbf{b}_{i+1}+\mathbf{b}_{i} \times \hat{\mathbf{e}}_{i+1}+\hat{\mathbf{e}}_{0} \times\left(\mathbf{b}_{i}-\mathbf{b}_{i+1}\right)\right]+O\left(h^{2}\right)\right\} \\
& =\mathbf{e}_{0}+2 h \sum_{i=1}^{4}\left[\left(\hat{\mathbf{e}}_{0}+\hat{\mathbf{e}}_{i}+\hat{\mathbf{e}}_{i+1}\right) \times\left(\mathbf{b}_{i}-\mathbf{b}_{i+1}\right)\right]+O\left(h^{2}\right)
\end{aligned}
$$




$$
=\mathbf{e}_{0}+2 h \sum_{i=1}^{4}\left[\left(\hat{\mathbf{e}}_{0}+\hat{\mathbf{e}}_{i}+\hat{\mathbf{e}}_{i+1}\right) \times \mathbf{a}_{i}\right]+O\left(h^{2}\right) .
$$

When this expression is substituted into the integral and expanded to order $h$, we obtain the result:

$$
\oiint_{\delta V} d \mathbf{S} \cdot \hat{\mathbf{n}}=\frac{h}{2} \hat{\mathbf{e}}_{0} \cdot \sum_{i=1}^{4}\left[\left(\hat{\mathbf{e}}_{i}+\hat{\mathbf{e}}_{i+1}\right) \times \mathbf{a}_{i}\right]+O\left(h^{2}\right) .
$$

Similarly, let us compute the volume of the enclosed figure. It consists of four triangular pillboxes, each composed of three tetrahedrons, so the volume can again be expressed in the form:

where

$$
V=V_{A}+V_{B}+V_{C}
$$

$$
\begin{aligned}
& 6 V_{A}=\sum_{i=1}^{4}\left[h \hat{\mathbf{e}}_{0} \cdot\left(\mathbf{b}_{i} \times \mathbf{b}_{i+1}\right)\right], \\
& 6 V_{B}=\sum_{i=1}^{4}\left\{h \hat{\mathbf{e}}_{i} \cdot\left[\left(\mathbf{b}_{i}-\mathbf{b}_{i+1}\right) \times\left(h \hat{\mathbf{e}}_{i}+\mathbf{b}_{i}\right)\right]\right\},
\end{aligned}
$$

and

$$
6 V_{C}=\sum_{i=1}^{4}\left\{h \hat{\mathbf{e}}_{i+1}\left[\left(\mathbf{b}_{i}^{\prime}-\mathbf{b}_{i+1}^{\prime}\right) \times \mathbf{b}_{i+1}^{\prime}\right]\right\} .
$$

These expressions may be expanded and combined to yield the expression:

$$
V=\frac{1}{6} h \sum_{i=1}^{4}\left[\left(\hat{\mathbf{e}}_{0}+\hat{\mathbf{e}}_{i}+\hat{\mathbf{e}}_{i+1}\right) \cdot \frac{1}{2}\left(\mathbf{a}_{i} \times \mathbf{a}_{i+1}\right)\right]+O\left(h^{2}\right) \text {. }
$$

The extra factor of $1 / 2$ arises from the conversion of the $b$-type vectors to the a-type vectors. Consequently, in limit as $h \rightarrow 0$ the ratio becomes:

$$
2 J=\frac{\hat{\mathbf{e}}_{0} \cdot \sum_{i=1}^{4}\left[\frac{1}{2}\left(\hat{\mathbf{e}}_{i}+\hat{\mathbf{e}}_{i+1}\right) \times \mathbf{a}_{i}\right]}{\sum_{i=1}^{4}\left[\frac{1}{3}\left(\hat{\mathbf{e}}_{0}+\hat{\mathbf{e}}_{i}+\hat{\mathbf{e}}_{i+1}\right) \cdot \frac{1}{4}\left(\mathbf{a}_{i} \times \mathbf{a}_{i+1}\right)\right]} .
$$

This formula has the same structure as the one we obtained earlier for a nodal point. The numerator represents a sum of approximations to line integrals around each of the four triangular patches which "fill" the quadrilateral. Consider the sum:

$$
\frac{1}{2}\left(\hat{\mathbf{e}}_{i}+\hat{\mathbf{e}}_{i+1}\right) \times \mathbf{a}_{i}+\frac{1}{2}\left(\hat{\mathbf{e}}_{i+1}+\hat{\mathbf{e}}_{0}\right) \times \mathbf{b}_{i+1}+\frac{1}{2}\left(\hat{\mathbf{e}}_{0}+\hat{\mathbf{e}}_{i}\right) \times\left(-\mathbf{b}_{i}\right),
$$


which consists of an average "normal" vector crossed into the associated line segment separating the two normals and directed in the "positive" direction. This expression can be rearranged and summed over all the elements surrounding the central node. Only the contribution from the first term survives, and this is precisely summation in the numerator of the above formula because the summation over $\hat{\mathbf{e}}_{0} \times \mathbf{a}_{i}$ makes no contribution. In the same spirit, the denominator represents the projected area of each triangular patch in the direction of the average normal, $\frac{1}{3}\left(\hat{\mathbf{e}}_{0}+\hat{\mathbf{e}}_{i}+\hat{\mathbf{e}}_{i+1}\right)$, composed of the unit normals at its vertices. All this is consistent with the alternative general expression for the mean curvature:

$$
2 J \hat{\mathbf{n}}=\lim _{S \rightarrow 0} \frac{1}{S} \oint_{C} \hat{\mathbf{n}} \times d \mathbf{r},
$$

where the integration is taken in the positive direction along the simple closed curve, $C$, which bounds an open, two-sided surface with area, $S$. Our formula for the mean curvature represents the discreet realization of this integral theorem. It be recast into even closer correspondence with this general expression by "factoring" out the vector, $\hat{e}_{0}$, (to within an additive contribution perpendicular to this unit vector). The result is

$$
2 J \hat{\mathbf{e}}_{0}=\frac{\sum_{i=1}^{4}\left[\frac{1}{2}\left(\hat{\mathbf{e}}_{i}+\hat{\mathbf{e}}_{i+1}\right) \times \mathbf{a}_{i}\right]}{\sum_{i=1}^{4}\left[\frac{1}{3}\left(\hat{\mathbf{e}}_{0}+\hat{\mathbf{e}}_{i}+\hat{\mathbf{e}}_{i+1}\right) \cdot \frac{1}{4}\left(\mathbf{a}_{i} \times \mathbf{a}_{i+1}\right)\right]}
$$

in which it is to be noted that the right-hand side again represents the ratio of two approximations, one for the line integral and the other for the enclosed area. The extra factor of $1 / 2$ in the denominator occurs because the actual area of the quadrilateral is counted twice in this particular construction.

It is of interest to note that this formula will give a non zero result even if the quadrilateral is not warped. All that is required is that adjacent quadrilaterals are not coplanar. Then there will be a "roof-like" structure at each vertex of the quadrilateral and an associated composite unit vector that is not parallel to the normal associated with the quadrilateral. The particular construction was chosen so as to obtain an expression which would remain valid as the meshes were made finer and finer. However, this objective was achieved after having chosen to associate with the warped quadrilateral element a normal direction through a particular point not necessarily located on the actual surface. 
Some questions still remain. Does the result we have obtained truly represent the mean curvature of a warped quadrilateral? Is it still possible for the mean curvature to vanish? It certainly is possible to imagine a smooth surface connecting the four nodes of a warped quadrilateral. Such a surface could very well have principal curvatures of opposite sign which add to zero. This behavior would signify the presence of a saddle. In order to investigate this possibility, it would by useful to also have available the second, or Gaussian, curvature of the surface. If we identify the principal curvatures at a point on a surface by the symbols, $k_{1}$ and $k_{2}$, then from the general expressions:

$$
2 J=k_{1}+k_{2} \text { and } K=k_{1} k_{2} \text {, }
$$

it follows that these principal curvatures can be computed from the formulas:

$$
k_{1}=J-\sqrt{J^{2}-K} \text { and } k_{2}=J+\sqrt{J^{2}-K} \text {, }
$$

where we have arbitrarily chosen $k_{1}$ to be the less positive value (which could be zero or negative). This motivates us to examine the calculation and implementation of the Gaussian curvature in part II of this study.

\section{Acknowledgment}

This particular study grew out of discussions with Dr. Janet Jones-Oliveira and Dr. Mark Christon (both of LLNL) and with Dr. Hans Fischer (U. Mass) and has been supported by the Laboratory's LDRD/ISR Program in Structural Acoustics under the direction of Dr. Terry Donich.

\section{References}

1 C. E. Weatherburn, Differential Geometry of Three Dimensions, (Cambridge University Press, 1961), Vol. I, pp. 226, 261.

2 M. R. Spiegel, Vector Analysis, (Schaum Publishing Company, N. Y., 1959), p. 123.

3 C. E. Weatherburn, op. cit., p. 240.

4 J. J. Koenderink, Solid Shape, (MIT Press, Cambridge, 1990), p. 217.

5 L. Brand, Vector and Tensor Analysis, (John Wiley \& Sons, NY, 1947), p. 14.

6 W. Gellert, S. Gottwald, M. Hellwich, H. Kästner, and H. Küstner, Eds., The VNR Concise Encyclopedia of Mathematics, 2nd ed., (Van Nostrand Reinhold, N. Y., 1989), pp. 298-299. 

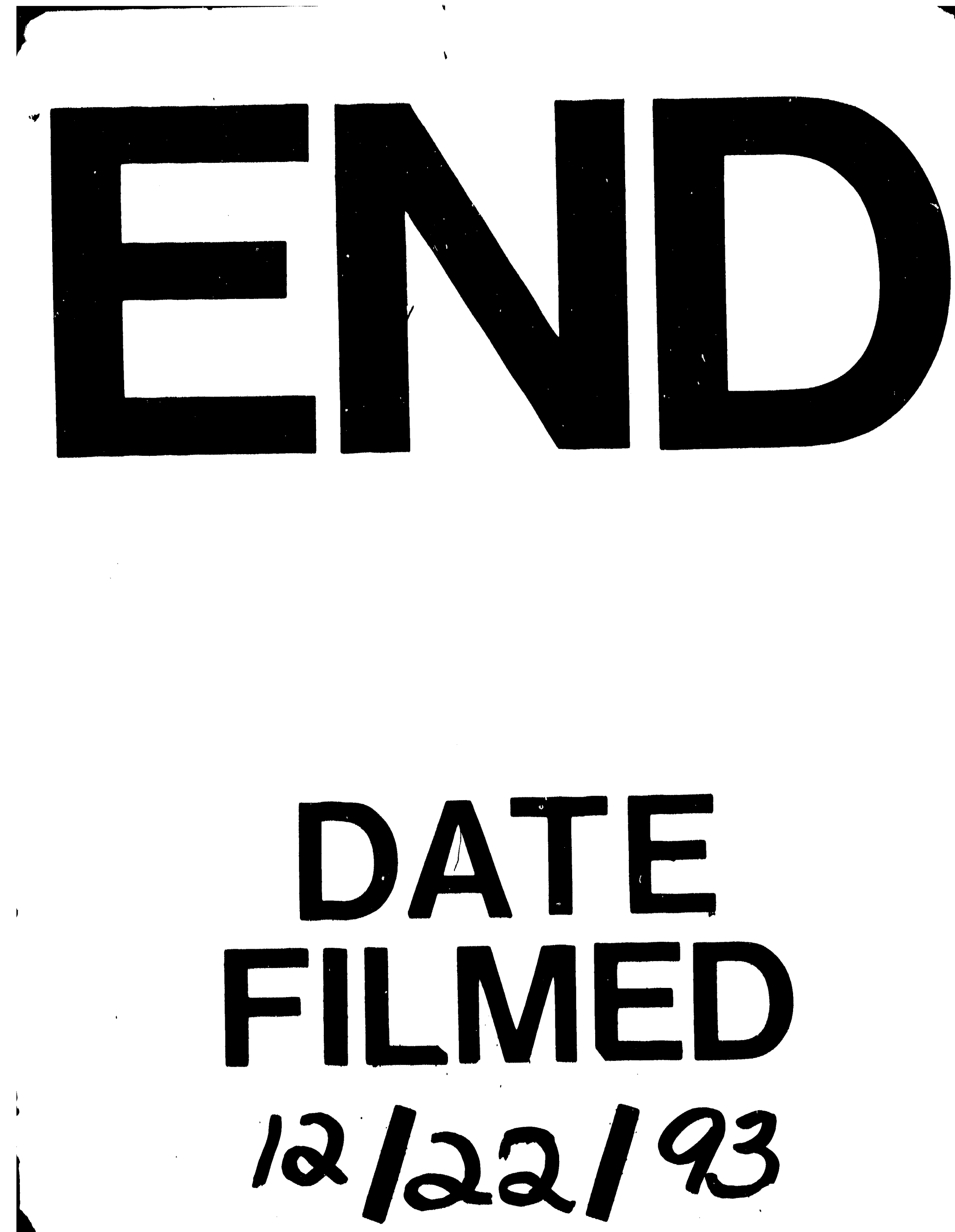

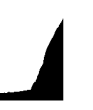


